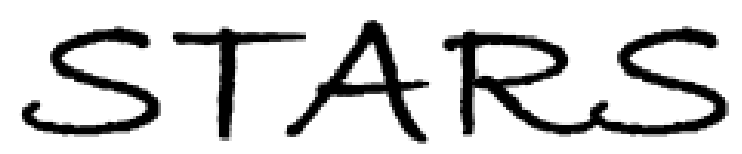

University of Central Florida

STARS

Faculty Bibliography 2000s

Faculty Bibliography

$1-1-2006$

\title{
Near-field characterization of effective optical interfaces
}

\author{
A. Apostol \\ University of Central Florida \\ D. Haefner \\ University of Central Florida
}

A. Dogariu

University of Central Florida

Find similar works at: https://stars.library.ucf.edu/facultybib2000

University of Central Florida Libraries http://library.ucf.edu

This Article is brought to you for free and open access by the Faculty Bibliography at STARS. It has been accepted for inclusion in Faculty Bibliography 2000s by an authorized administrator of STARS. For more information, please contactSTARS@ucf.edu.

\section{Recommended Citation}

Apostol, A.; Haefner, D.; and Dogariu, A., "Near-field characterization of effective optical interfaces" (2006). Faculty Bibliography 2000s. 5909.

https://stars.library.ucf.edu/facultybib2000/5909

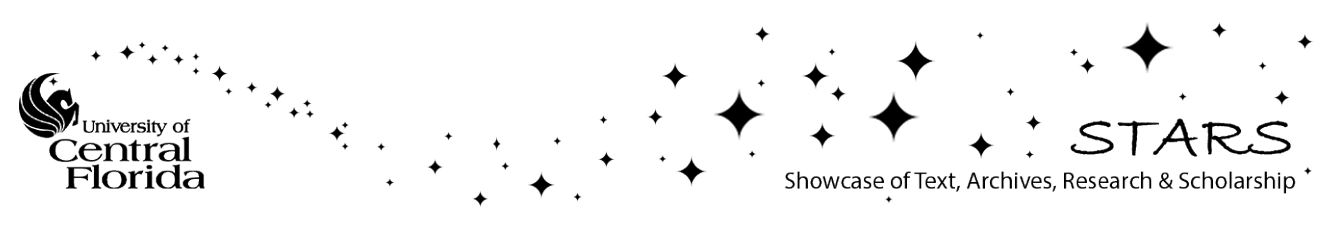




\title{
Near-field characterization of effective optical interfaces
}

\author{
A. Apostol, D. Haefner, and A. Dogariu \\ CREOL, College of Optics and Photonics, University of Central Florida, Orlando, Florida 32816-2700, USA
}

(Received 9 June 2006; published 7 December 2006)

\begin{abstract}
The properties of many heterogeneous media depend on both the surface roughness and the local variations of the optical properties. An effective optical interface is usually invoked to describe the characteristics of such media. Using approaches specific to near-field optics, the two influences can be decoupled and a quantitative assessment of their contributions can be performed. It is also shown that a discrete random-walk model can be used to determine the magnitude of the dielectric constant fluctuations at subwavelength scales which, in turn, describe the morphology of optically inhomogeneous media.
\end{abstract}

DOI: 10.1103/PhysRevE.74.066603

PACS number(s): 42.25.Kb, 42.50.Ar, 07.79.Fc

\section{INTRODUCTION}

Optical properties of material systems are commonly described in terms of their dielectric function. For optically homogeneous materials, the dielectric function determines the refractive index and the absorption properties at specific optical frequencies. In practice however, many composite materials are such that their dielectric functions vary randomly with position. When the spatial scale of the heterogeneities is much smaller than the wavelength of light, "effective-medium" approaches can be applied and a macroscopic effective permittivity can be attributed to the medium [1].

For complex cases such as particulate media, defining an effective dielectric function is not straightforward. First, the situation is complicated by the fact that the spatial scale of inhomogeneities is not much smaller than the wavelength and therefore, these materials scatter light significantly. Second, materials can have appreciable roughness at a scale comparable with the wavelength of light, which also impacts the scattering properties. As a result, the macroscopic optical properties of a composite medium depend not only on the bulk heterogeneities (composition and sizes of particulates, for instance) but also on the surface roughness generated by the particles themselves. In other words, for a complete characterization of such random media, both optical and structural considerations must be taken into account. To accomplish this task one must be able to decouple the impact of surface roughness on the overall optical characteristics from the influence of the bulk properties.

Conventional optical measurements are usually performed in the far field and depend on both bulk and surface properties of optically inhomogeneous media making the design of the overall optical properties a daunting task. Accordingly, many of the methods employed today for predicting the optical properties of the composite materials rely on phenomenological models [2-4]. It can be envisioned however, that studying the optical phenomena in the proximity of the physical interface might permit discrimination between the two major contributions to the overall optical properties. One could, subsequently, use propagation models to predict the far-field properties.

The approach described in this paper is to employ nearfield optics instrumentation for determining simultaneously mechanical and optical properties at the physical interface. In general, the surface of a composite medium can manifest fluctuations of both the value of local dielectric function and the interface topography. This is a complex situation where the optical constants at subwavelength scales combine with the physical roughness to determine the optical properties of the radiation emitted or scattered from such a medium. In general, one could regard these optical properties as being determined by an "effective optical interface." In this paper we will use a statistical treatment that allows differentiation between these two contributions to the effective optical interface. Specifically, we will discuss comparatively the influence of surface roughness for different diffusive and nonabsorbent materials, which are also locally inhomogeneous in terms of their refractive indices. It will be demonstrated that the statistical properties of the surface roughness and those of the subsurface variations of the local dielectric constant can be determined independently.

\section{NEAR FIELD OF HETEROGENEOUS MEDIA}

The radiative properties of materials with significant variations of their optical constants are dominated by scattering. The interaction between optical waves and such randomly inhomogeneous media has been systematically investigated and it is now well understood that the familiar appearance of speckle patterns can be described in terms of Gaussian and non-Gaussian statistics of light fluctuations. While far-field intensity variations have been studied in detail for quite some time [5], it was only recently demonstrated that the presence and the confinement of the evanescent waves in the near field of the random media may affect the statistical properties of optical fields in the vicinity of the interfaces [6-8].

A speckle pattern is produced by interference between many scattering waves having random amplitudes and phases. If the amplitudes and the phases of the field components are statistically independent random variables originating from a large number of elementary scattering centers and if the phases are uniformly distributed, then the real and imaginary parts of the complex scattered fields have circular Gaussian distributions. Consequently, the amplitude of the resultant field has a Rayleigh distribution; the corresponding intensity is exponentially distributed and the optical contrast, 


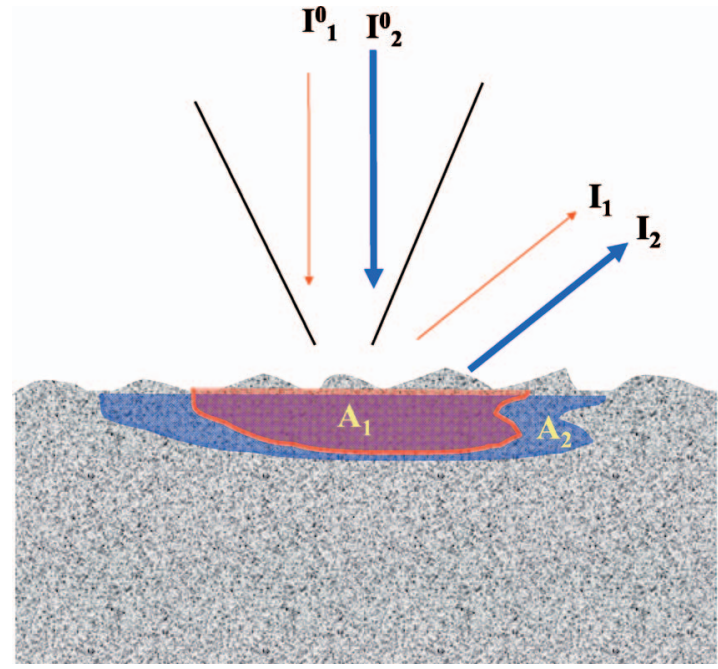

FIG. 1. (Color) The coupling of the incident radiation with different intensities $I_{2}^{0}>I_{1}^{0}$ into an optically inhomogeneous medium and its subsequent reemission. Due to an inherent cutoff of the intensity detection system, the emitted radiation is collected from a larger area $A_{2}>A_{1}$ when the value of the incident intensity increases.

i.e., theintensity variance normalized by the average intensity, equals unity.

When there is a certain correlation between the superposing waves, which form a speckle pattern, the statistics of resultant field distribution has a non-Gaussian behavior. A similar situation occurs when the total field is the result of the superposition of a reduced number of independent contributions. This regime is generated when, at a given observation point, the field is not a superposition of a large number of contributions originating from different locations within the random medium. Non-Gaussian statistics may also be the result of weak scattering, when the phases of the elementary scattering centers are not uniformly distributed anymore and their probability density function depends on the surface statistics.

Another situation resulting in a non-Gaussian regime of scattering is when a random medium is illuminated through the tip of a near-field scanning optical microscope [9]. This is illustrated in Fig. 1, where the radiation is coupled onto a medium, which has subwavelength variations in both the topography and its optical properties, and is then reemitted in the form of scattered field. In practice, light from a laser source is coupled to one end of an optical fiber and it emanates from the other, which has been tapered down to an aperture of $100 \mathrm{~nm}$ and it is $\mathrm{Al}$ coated. The fiber couples both propagating and evanescent waves onto the sample and, during the scattering process, some of the evanescent components are being converted into homogeneous waves, which are then detected in the far field by an avalanche photodiode. The near-field scanning optical microscope (NSOM) probe has the possibility to scan in constant distance mode and to simultaneously obtain the atomic force microscopy (AFM) topography and the near-field optical images with a resolution well below the wavelength of light. A typical topographical image and its corresponding near-field intensity
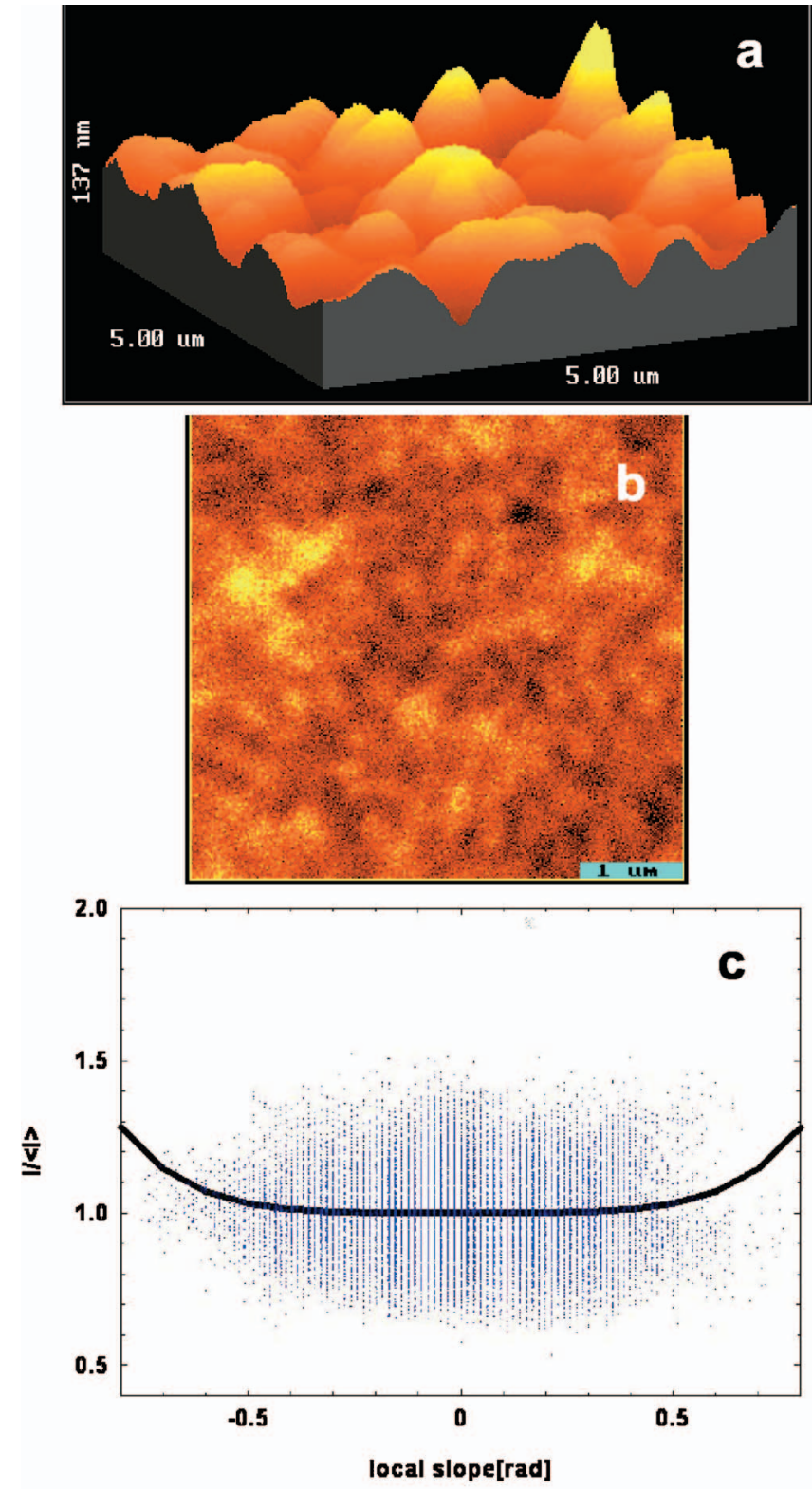

FIG. 2. (Color) (a) Topography of compact slabs made of calcium carbonate and kaolin microparticles; the rms roughness is $22.7 \mathrm{~nm}$. (b) Near-field intensity distribution collected over the same area. (c) Dependence of the normalized near-field intensity on the local slope. The continuous curves are the theoretical predictions based on a model using the Fresnel reflection coefficients evaluated for an effective index of refraction of $n=1.5$.

distribution are presented in Figs. 2(a) and 2(b). The data was collected on an optical composite (coatings) made of calcium carbonate and kaolin microparticles. The composite layers examined here had typical thicknesses of several hundred microns and rms roughness of the order of $\lambda / 20$.

When the $100 \mathrm{~nm}$ aperture tip scans across the surface of the sample, the coupling of radiation onto the medium is a complex process such that the magnitude of the reemitted radiation depends not only on the local values of the dielectric constant but also on the state of the physical interface. To better illustrate this complex situation, we present in Fig. 2(c) a typical dependence of the normalized detected 
intensity on the local slope of surface. Such a histogram can be generated taking advantage of the fact that both the scattered intensity and the topographical data are recorded simultaneously during the NSOM scan.

The local slopes of the surface have been determined from the topographical data, by sampling the heights with a lateral or vertical step equal to the size of the tip. Also shown with a continuous curve in Fig. 2(c) is the reflectance predicted by the Fresnel coefficients evaluated for an optically homogeneous medium with an effective permittivity as estimated using the conventional Maxwell-Garnett mixing rule. One can clearly see that this approach fails completely in describing the dependence of the coupling coefficient on both the local slope and the refractive index variations. Because of strong local inhomogeneities, the fluctuations of the dielectric constant will be accounted for in a discrete scattering model that will be described later.

Before proceeding to any quantitative analysis, we would like to emphasize that the histogram of Fig. 2(c) synthesizes all of the topographical and intensity information presented in Figs. 2(a) and 2(b), respectively. The distribution of local slopes, more precisely, the longitudinal spread of the histogram, describes the topographical character of the interface, accounting for both the rms roughness and lateral correlation length of the surface profile. On the other hand, the intensity fluctuations determined by the local optical permittivity can be regarded as the vertical spread of the histogram. Therefore, this representation relates the local variation of the dielectric constant to the morphology of the physical interface, offering a comprehensive, yet qualitative, description of the effective optical interface of a heterogeneous material. As it will be shown in the following, a statistical treatment is necessary for decoupling the optical and topographical contributions to the effective optical interface.

\section{A. Random-walk model of scattering}

As can be seen in Fig. 2(b), the spatial distribution of the detected intensity resembles the typical intensity variations of a speckle pattern. The intensity probability density function is well approximated by a $\Gamma$-function while the contrast takes values significantly smaller than unity. Interestingly, the intensity probability density function, and therefore the optical contrast, depends on the measured average intensity, which is adjusted by changing the incident intensity coupled into the fiber tip. Such a dependence of the statistical properties of the scattered field on the excitation level illustrated in Fig. 1, is somewhat puzzling because one should not expect any nonlinear phenomena in the media examined. Details about this behavior have been published previously [8], and can be briefly explained by noting that, the radiation couples onto the medium away from the tip before being reemitted and captured in the far field by the detection system. More importantly, one should realize that the noise level of the light detection process imposes an inherent intensity cutoff. As a result, when the intensity coupled into the medium increases, the detection system effectively collects radiation from a larger area on the surface of the medium.

To account for these observations, a simple model can be developed by considering that, due to the variations of the optical properties, the light is collected from the medium over a surface of area $A$, the size of which depends on the incident intensity, as suggested in Fig. 1. Within this area, $\mathrm{N}$-independent elementary scattering centers are being excited. In a first approximation, one can further consider that $A$ is proportional with the incident intensity, and then so is $N$. In this description, $N$ represents a number of "equivalent scattering centers" distributed across the physical interface of the random medium.

Reference [8] contains details about this model, which we summarize here. First, due to the complexity of the process of radiation coupling onto the medium, the elementary scattering centers are not excited in phase. However, one can assume that the distribution of their initial phase $\theta_{i}$ can be considered to be a Gaussian function with the rms fluctuations $\Delta \theta_{i}$ determined by the local properties of the medium. Second, due to surface topography, the radiation reemitted by the elementary scattering centers accumulates an additional phase $\theta_{p}$, which is Gaussian distributed with a width $\Delta \theta_{p}$ determined by the distribution of the surface heights. Therefore, the probability density function of the total phase, $\theta=\theta_{i}+\theta_{p}$, is approximated by a Gaussian distribution with the rms phase fluctuations $\Delta \theta=\sqrt{\Delta \theta_{i}^{2}+\Delta \theta_{p}^{2}}$, determined by both surface $\left(\Delta \theta_{p}\right)$ and subsurface $\left(\Delta \theta_{i}\right)$ properties of the inhomogeneous medium.

The far-field radiation can be now regarded as a coherent superposition of waves scattered by the $N$-independent elementary scattering centers and can be described as a random walk in the complex space. When these scattered waves have equal amplitudes $a$, their coherent superposition forms a speckle pattern whose intensity statistics depend only on the number $N$ and the phase distribution $\Delta \theta[10,11]$. A typical outcome of this model is illustrated in Fig. 3(a) where the optical contrast is plotted as function of the normalized average intensity. This dependence was evaluated for different phase distributions of the scattering centers and the normalization constant represents the intensity contribution of one elementary contribution $I(1)=a^{2}$. The calculations were performed for phase distributions, which have $\Delta \theta_{1}=0.5 \mathrm{rad}$ and $\Delta \theta_{2}=2 \mathrm{rad}$, respectively, and are also shown in Fig. 3(b). These values of rms phase fluctuations are representative for the two experimental situations, which will be discussed in the following.

As depicted in Fig. 3(a), the random-walk model evaluated for the case of nonuniform phase distribution and a small number of scattering centers $N$, predicts a decrease of the optical contrast with $N$, and therefore, with the average intensity $\langle I\rangle$. One can also notice that, the broader the phase distribution of the elementary scattering centers, the larger the values of the speckle contrast.

\section{B. Quantitative description of effective optical interfaces}

Systematic studies have been conducted for media with inhomogeneous refractive indices along the surface in situations where the dielectric constant varies over scales smaller than the wavelength of optical radiation. The results reported here refer to two kinds of inhomogeneous media, which have quite different optical properties. 

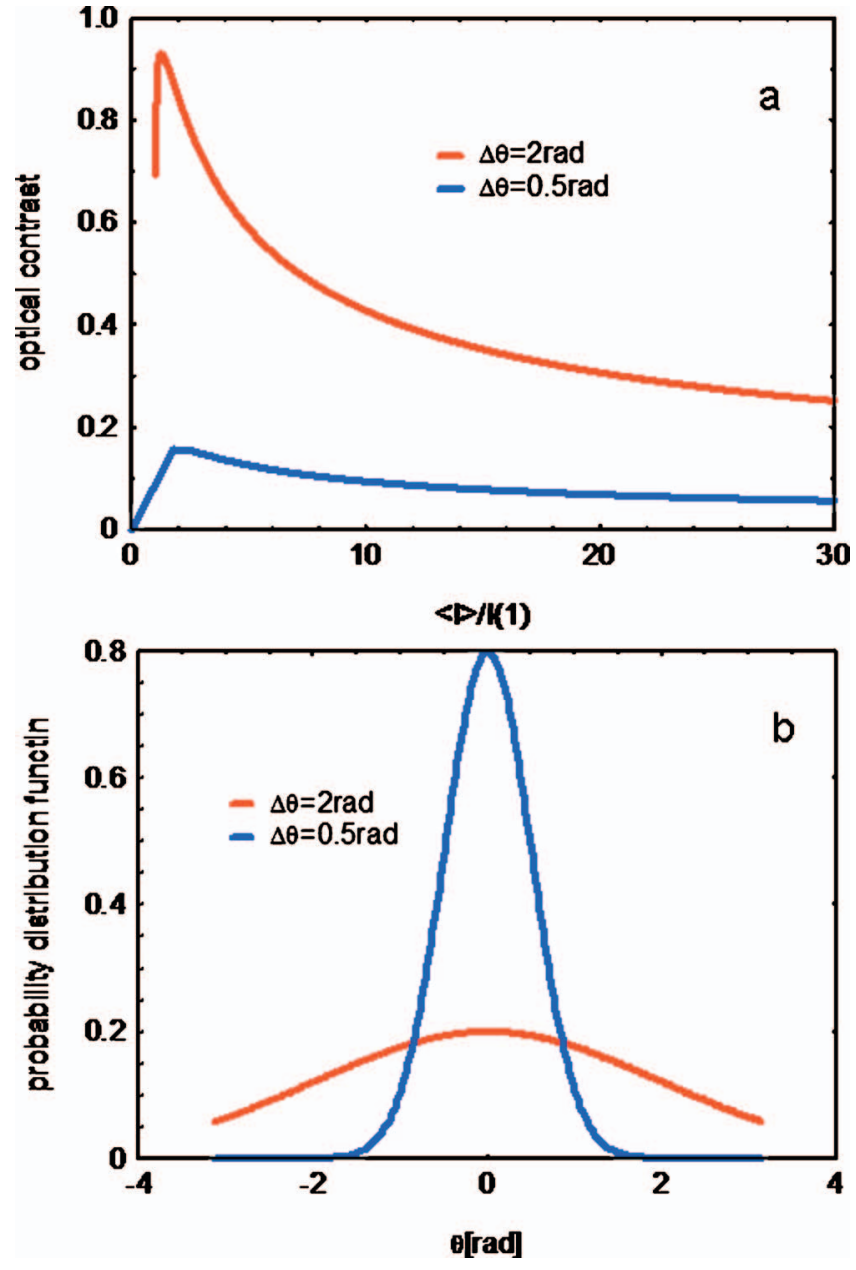

FIG. 3. (Color) (a) Optical contrast as a function of the average intensity for different values of the rms phase distribution as indicated. (b) Phase probability distribution function with different rms phase fluctuations $\Delta \theta$, as indicated.

The first type of samples are slabs made of calcium carbonate and kaolin microparticles, which were compacted such that they generate pores with a size distribution centered at $20 \mathrm{~nm}$ as determined by the mercury porosimetry [12]. These media are optically opaque, their thickness is $1 \mathrm{~mm}$, and the refractive index has small variations centered around $n=1.5$. The rms fluctuations of the surface height range between $\lambda / 40$ and $\lambda / 10$ while the lateral correlation length of the interface heights is comparable with the wavelength of light.

The second type of inhomogeneous media examined were optical coatings containing 100 parts latex and 25 parts $\mathrm{TiO}_{2}$ particulates. In these samples the uniform distribution of the pigment was proven by scanning electron microscopy (SEM) and energy dispersive x-ray spectroscopy (EDS) analysis. In comparison with the first type of samples, the index of refraction now has a much broader distribution ranging between $n=1.5$ and $n=3.2$. The rms fluctuations of the surface heights vary between $\lambda / 10$ and $\lambda / 6$ but, in this case, the lateral correlation length of surface profile is much larger than the wavelength of light

Using the illumination procedure described before, NSOM scans were performed and both topographical and

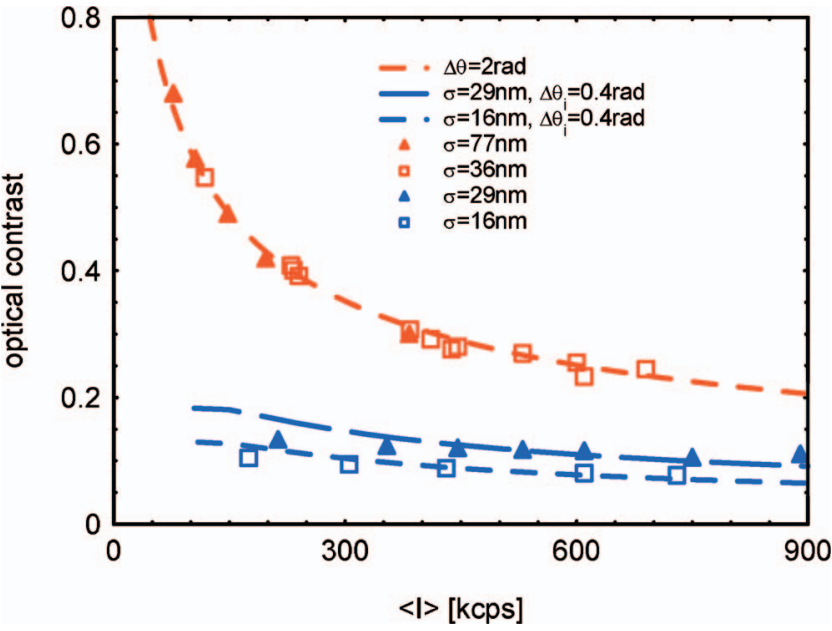

FIG. 4. (Color) Dependence of the optical contrast on the average intensity. The symbols represent typical experimental results corresponding to different surface roughness as indicated, of the compact slabs of calcium carbonate and kaolin microparticles (first type media: blue symbols) and of the optical coating containing 100 parts Latex and 25 parts $\mathrm{TiO}_{2}$ particulates (second type media: red symbols). The curves are the theoretical predictions based on the random-walk model of Refs. [10,11] evaluated for $\Delta \theta_{i}=0.4 \mathrm{rad}$ (blue curves) and $\Delta \theta=2 \mathrm{rad}$ (red curve).

scattered intensity data were recorded for different values of the excitation intensity. The fluctuating intensity maps were subsequently processed and experimental values of the optical contrast and of the average intensity are presented in Fig. 4 for the two typical samples having different surface roughness. The data were recorded over areas of $5 \times 5$ microns situated at the same distances from the surface.

The random-walk model outlined above makes a good description of the experimental results. We should emphasize that the number $N$ of the elementary scattering centers increases when the excitation level increases, and so does the average intensity $\langle I\rangle$ over the scanning area. However, the phase distribution of the scattering centers is not expected to change. This is a consequence of the fact that the effective local properties of the medium are the same irrespective of the size of the illuminated area.

The continuous curves represent the results of calculations using the random-walk model that best describe the experimental data. The values of the rms fluctuations $\Delta \theta$ of the total phases as inferred from this fit were the ones used in the illustration of Fig. 3. As can be seen, the uniform distribution of the $\mathrm{TiO}_{2}$ pigment in the latex matrix leads to a broad initial phase distribution of the elementary scattering centers [depicted by the red curve in Fig. 3(b)] and, therefore, to larger values of the optical contrast as represented by the red symbols in Fig. 4. On the other hand, due to smaller variations in the local refractive indices, the compact slabs made of calcium carbonate and kaolin microparticles are characterized by a rather narrow phase distribution [depicted by the blue curve in Fig. 3(b)]. Consequently, in this case the intensity fluctuations are much smaller, i.e., lower values of optical contrast as illustrated by the blue symbols in Fig. 4. It is now clear that in the case of the locally inhomogeneous 
media, the optical contrast is a measure of the intensity fluctuations and relates to the fluctuations of the dielectric constant.

In order to quantify the differences introduced by the variations of the dielectric constant, one needs to take into account the statistical properties of the physical interface. First, we should emphasize that in our experimental situations the surface statistics is stationary over areas of size $A$. This is happening when the size of the excited regions is comparable to the lateral correlation area of the surface, as in the case of the compact slabs of calcium carbonate and kaolin microparticles. In these circumstances, one can consider that, at each scanning step, areas of size $A$ have the same surface statistics described by a Gaussian height distribution with $\sigma$ rms fluctuations. The rms fluctuations of the propagating phase are determined by the surface roughness, $\Delta \theta_{p}$ $=k_{o} \sigma$, where $k_{o}$ is the wave number in air and $\sigma$ represents the rms height of the surface fluctuations. As the distribution of interface heights is Gaussian, so will be the additional phases $\theta_{p}$ of the emitted field. Therefore, each blue curve in Fig. 4 is evaluated using the model in Ref. [10] for Gaussian probability distributions of the phase having width $\Delta \theta$ $=\sqrt{\Delta \theta_{i}^{2}+\Delta \theta_{p}^{2}}$, where $\Delta \theta_{p}=k_{o} \sigma$ with $\sigma$ being the measured roughness of the scanned area. The values $I(1)=60 \mathrm{kcps}$ and $\Delta \theta_{i}=0.4 \mathrm{rad}$ are found to be the best fit for all our experimental data corresponding to surfaces with different rms height fluctuations. We can conclude that the value $\Delta \theta_{i}$ $=0.4 \mathrm{rad}$ of the initial rms phase fluctuations should be determined by the local variations of the dielectric constant and it is therefore material dependent.

Let us now take a closer look at the dependence of the optical contrast on the average intensity for the first type media, which is shown in Fig. 4. Surfaces with different roughness were considered both experimentally (blue symbols) and theoretically (blue curves). The reported data correspond to different values of the rms roughness: $\sigma_{1}=16 \mathrm{~nm}$ and $\sigma_{2}=29 \mathrm{~nm}$ as indicated. One can clearly see that, as expected, the rougher the scanned surface, the larger the values of the speckle contrast because an increase in the additional phases $\theta_{p}$ leads eventually to a broader distribution of phase and consequently to a higher contrast value.

A rather different physical situation is encountered in the case of the second type of optical coatings containing latex and $\mathrm{TiO}_{2}$ particulates. Here, the areas of size $A$ are much smaller than the lateral correlation area of the surface. The stationarity condition for the surface statistics is again fulfilled as a flat surface is practically probed at each scanning step. In other words, at each pixel of the NSOM image, the illumination area is mostly on a horizontal plane and the rms fluctuations of the propagating phase $\Delta \theta_{p}$ are essentially zero irrespective of the measured surface roughness. Accordingly, the variations of the total phase $\theta$ are practically determined only by the initial phase distribution of the exciting effective scattering centers, i.e., $\Delta \theta=\Delta \theta_{i}$. The red symbols in Fig. 4 represent the dependence of the optical contrast on the average intensity for two different values of surface roughness: $\sigma_{1}=36 \mathrm{~nm}$ and $\sigma_{2}=77 \mathrm{~nm}$. As can be seen, the influence of the rms roughness is negligible in this case because of the large values of the lateral correlation length. The best fit to the data corresponds to $I(1)=20 \mathrm{kcps}$ and $\Delta \theta=\Delta \theta_{i}=2 \mathrm{rad}$. This broadening of the phase distribution is determined by the larger average fluctuations of the dielectric function in the case of high refractive index $\mathrm{TiO}_{2}$ pigment embedded in the latex matrix.

The results presented here demonstrate that a broader distribution of the refractive index along an optically inhomogeneous interface determines larger values of the near-field intensity fluctuations. Moreover, the simultaneous acquisition of the surface heights together with the optical properties allows us to account quantitatively for the effect of the interface statistics (measured by $\sigma$ ) on the rms fluctuations of the total phase $\Delta \theta$. Therefore, the variations of the initial phase distribution $\Delta \theta_{i}$ can be determined and subsequently related to the fluctuations of the local dielectric constant along the interface.

Our findings are also supported by a numerical simulation using the coupled dipole approximation (CDA). This numerical approach approximates a continuum volume with a finite array of polarizable points [13]. These discrete dipoles react to the local field and, by the use of dyadic Green's function, the complete interactions between dipoles, including both the near- and far-field components, are accounted for. The polarizability of each individual dipole relates to the local dielectric properties of the sample. The initial excitation is produced by an emitting dipole, which is placed in the near field of the medium and simulates the tip of the fiber. The far-field coherent superposition of the dipolar contributions is recorded for specific configurations of the scattering medium and an ensemble of realizations is generated.

To model the two composite materials discussed here, dipoles having the polarizabilities given by the Lorenz-Lorenz formula corresponding to the specific components were randomly distributed in a proportion according to the sample's composition. A large number of realizations of a sample is generated and the relevant statistical information regarding the phase, amplitude, and intensity distributions are determined. The specific details of this procedure will be presented elsewhere [14].

The CDA numerical simulations provided a full description of the amplitude and phase distributions corresponding to different realizations of random media. It has been consistently observed that a greater refractive index contrast yields a broader distribution of phases for contributions of different elementary scattering centers. In addition, for the particular case of the two materials examined here, the ratio between optical contrasts calculated for two different values of the average intensity such that $\langle I\rangle_{1} /\langle I\rangle_{2}=2$, was 1.63 and 1.17 for $\mathrm{TiO}_{2}$ and Mylar samples, respectively. Using the data presented in Fig. 4, the corresponding ratios evaluated for the experimental data are 1.46 and 1.02 , respectively. Moreover, for these values of the average intensities, $\langle I\rangle_{1}$ and $\langle I\rangle_{2}$, we also evaluated the ratios between the optical contrasts for each type of medium. The CDA simulations predict values of 3.27 and, respectively, 2.34 for the $\mathrm{TiO}_{2}$ particulates and the calcium carbonate and kaolin microparticles, respectively. These are in good agreement with the ratio of 3.15 and, respectively, 2.19 obtained experimentally as shown in Fig. 4. The fact that the experimental results corroborate well 
with the numerical estimations proves that the associated direct-scattering problem can be handled properly using the CDA approach. In terms of modeling the physical phenomenon, the simple description in terms of a superposition of waves with random phases provides a good explanation for the phenomena observed in the near field of random media.

\section{CONCLUSIONS}

In conclusion, the optical properties of heterogeneous media depend on both the surface roughness and the variations of the local dielectric constant. We have demonstrated that the subwavelength scale fluctuations of the dielectric constant can be taken into account if the near-field intensity fluctuations are described using a discrete random-walk model. Typical information collected during a near-field optical scan allows for the surface statistics to be properly accounted for. Subsequent analysis permits differentiating between the topographical and optical contributions to the effective optical interface. This decoupling between the mechanical and the optical characteristics of the effective surface together with the stochastic properties of the scattered intensity can be used to obtain information about the local variations of the dielectric constant which, in turn, relate to the morphological properties of the inhomogeneous material.

Based on the coupled dipole approximation, we developed a numerical simulation procedure for examining the statistical properties of the radiation scattered by random media. This brought additional confirmation for the fact that the stochastic properties of the scattered radiation are directly related to variations of the optical properties of heteroge- neous media such as dispersions of pigment particles in a host material.

In our experimental configuration, we have seen that, during the illumination process, the radiation is first coupled into the medium before being reemitted and, therefore, a statistical treatment of the near-field intensity can offer new possibilities for subsurface characterization of inhomogeneous media. Potential diagnostics of surface and subsurface properties suggest promising applications in cell biology.

Surface variations can be a driver of overall optical properties and this is a degree of freedom, which was not previously recognized in designing diffuse optical coatings. By understanding and leveraging the ability to imbed fluctuations of the dielectric constant into the surface of a paper coating for instance, the optical properties can be controlled and enhanced to new levels.

Another relevant observation is worth making. To optimize materials characteristics, the detailed microscopic properties and the macroscopic responses of heterogeneous structures are usually examined with different tools. This is one of reasons why the advent of engineering composites (both solid or soft materials) is most of the time driven by empirical approaches. The method illustrated in this paper permits a direct and quantitative investigation of multiscale aspects of the dielectric response of random composite materials.

Finally, one should be aware that, in general, some of the electromagnetic responses of heterogeneous materials bear significant similarities with other physical properties such as thermal, mechanical, permeability, etc. In this respect, our results might be significant for understanding and decoupling phenomena determined by inhomogeneous interfaces.
[1] A. Pirou, Dielectric Properties of Heterogeneous Materials (Elsevier, New York, 1992).

[2] J. M. Elson, Phys. Rev. B 30, 5460 (1984).

[3] S. Mudaliar, Waves Random Media 4, 167 (1994).

[4] A. Sentac, H. Giovannini, and M. Saillard, J. Opt. Soc. Am. A 19, 727 (2002).

[5] Laser Speckle and Related Phenomena (J.C. Dainty, New York, 1975).

[6] A. Apostol and A. Dogariu, Opt. Lett. 29, 235 (2004).

[7] A. Apostol and A. Dogariu, Phys. Rev. Lett. 91, 093901 (2003)
[8] A. Apostol and A. Dogariu, Phys. Rev. E 72, 025602 (2005).

[9] K. Lieberman, N. Ben-Ami, and A. Lewis, Rev. Sci. Instrum. 67, 3567 (1996).

[10] Richard Barakat, J. Opt. Soc. Am. 71, 86 (1981).

[11] J. Goodman, Statistical Optics (John Wiley \& Sons, New York, 1985).

[12] J. Van Brakel, S. Modry, and M. Svata, Powder Technol. 29, 1 (1981).

[13] E. M. Purcell and C. R. Pennypacker, Astrophys. J. 186, 705 (1973).

[14] D. Haefner, A. Apostol, and A. Dogariu (unpublished). 